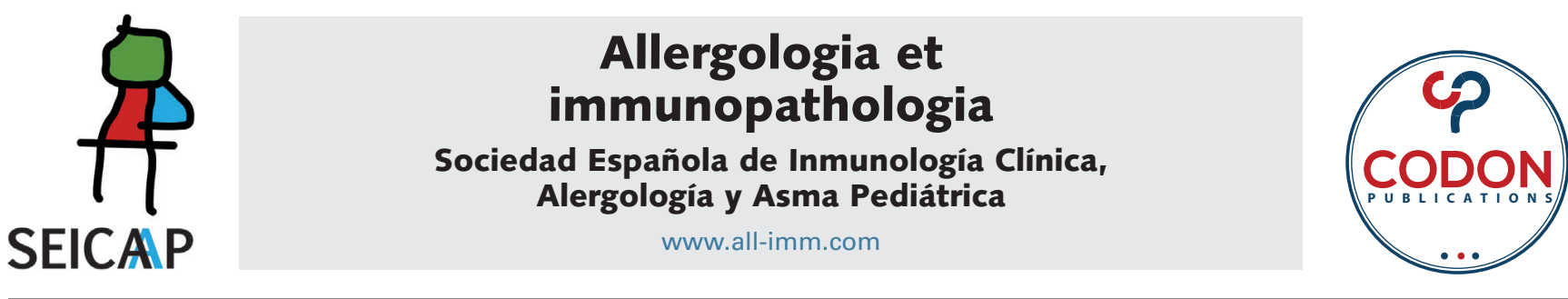

RESEARCH ARTICLE

\title{
PRMT5 promotes inflammation of cigarette smoke extract-induced bronchial epithelial cells by up-regulation of CXCL10
}

\author{
Junfang Ju, Yingchun $\mathrm{He}^{*}$
}

Department of Geriatrics, Hangzhou TCM Hospital Affiliated to Zhejiang Chinese Medical University, Hangzhou, Zhejiang Province, China

Received 22 July 2021; Accepted 13 August 2021

Available online 1 September 2021

\section{KEYWORDS \\ bronchial epithelial \\ cells; \\ chronic obstructive \\ pulmonary disease; \\ cigarette smoke \\ extract; \\ CXCL10; \\ inflammation; \\ PRMT5}

\begin{abstract}
Background: Chronic obstructive pulmonary disease (COPD) is related to inflammation and obstruction of the lungs and airways. Protein arginine methyltransferase 5 (PRMT5) that promotes arginine methylation of histones is associated with inflammation of endothelial cell and is implicated in lung branching morphogenesis and progression of lung cancer. The mechanism of PRMT5 in inflammatory response of COPD was explored in this study.

Methods: Human bronchial epithelial cells, $16 \mathrm{HBE}$, were treated with cigarette smoke extract for $24 \mathrm{~h}$ to establish cell model of COPD. Cell viability was examined by MTT assay. Western blot and reverse transcription-quantitative polymerase chain reaction (RT-qPCR) assays were used to explore expression of PRMT5. Expression of Interleukin (IL)-6, IL-8, tumor necrosis factor- $\alpha$ (TNF- $\alpha$ ), and IL-1 $\beta$ were investigated by enzyme-linked-ithe mmunosorbent serologic assay.

Results: Cigarette smoke extract treatment induced cytotoxity of $16 \mathrm{HBE}$ with reduced cell viability. PRMT5 was enhanced in cigarette smoke extract-induced 16HBE. Knockdown of PRMT5 increased cell viability of cigarette smoke extract-induced $16 \mathrm{HBE}$, and attenuated cigarette smoke extract-induced increase of IL- 6 , IL- 8 , TNF- $\alpha$, and IL- $1 \beta$. Up-regulation of C-X-C Motif Chemokine 10 (CXCL10) in cigarette smoke extract-induced 16HBE was restored by knockdown of PRMT5. Over-expression of CXCL10 counteracted with the suppressive effect of PRMT5 silence on expression of IL-6, IL-8, TNF- $\alpha$, and IL-1 $\beta$. Moreover, PRMT5 silence-induced increase of cell viability in cigarette smoke extract-induced $16 \mathrm{HBE}$ was reversed by over-expression of CXCL10.

Conclusion: Knockdown of PRMT5 promoted cell viability of cigarette smoke extract-induced $16 \mathrm{HBE}$, and reduced inflammation through down-regulation of CXCL10.

(c) 2021 Codon Publications. Published by Codon Publications.
\end{abstract}

*Corresponding author: Yingchun He, Department of Geriatrics, Hangzhou TCM Hospital Affiliated to Zhejiang Chinese Medical University, No. 453 Stadium Road, Xihu District, Hangzhou, Zhejiang Province 310007, China. E-mail address: yc_he1@163.com 


\section{Introduction}

Chronic obstructive pulmonary disease (COPD) is one of the most common respiratory diseases and is characterized by progressive and irreversible limitation of airflow. ${ }^{1}$ The most common pathological features of COPD are emphysema caused by small airway stenosis and rupture of the lung tissue that results in high morbidity and mortality. ${ }^{2}$ COPD is associated with exposure to harmful gases and particles that lead to abnormal inflammatory responses. ${ }^{1}$ Genetics and environment are the major factors for the etiology of COPD, and the most cases of COPD are caused by environment factors, especially chronic smoking. ${ }^{3}$ Cigarette smoking and other inhaled substances stimulate macrophages and epithelial cells to release large amounts of chemokines, and subsequently neutrophils, monocytes, and lymphocytes are recruited to the damaged tissues, such as peripheral airways and the lung parenchyma. ${ }^{4}$ Therefore, reducing exposure to risk factors, including smoking and exposure to second-hand smoke, is an effective way to prevent COPD. ${ }^{5}$

Protein arginine methyltransferase (PRMT) family catalyzes arginine monomethylation, asymmetric, or symmetrical dimethylation modifications, involved in conserved and ubiquitous physiological processes. ${ }^{6}$ Regulation of PRMTs on immune cells is related to pulmonary disorders. ${ }^{7}$ PRMT5 modulates cell growth and transformation through catalyzation of omega- $\mathrm{N}^{G}$-mono-methyl arginine and symmetric omega- $N^{G}$, and $N^{G}$-dimethyl arginine was shown to be associated with the progression of lung cancer. ${ }^{8}$ Cell metastasis of lung cancer was promoted by PRMT5. ${ }^{9}$ PRMT5 was also implicated in inflammatory diseases. ${ }^{10}$ For example, PRMT5 regulated differentiation and function of abstract regulatory $\mathrm{T}$ cells (Tregs) during the development of ulcerative colitis, ${ }^{11}$ and suppressed synovial destruction and inflammation to attenuate rheumatoid arthritis. ${ }^{11}$ Previous study has shown that the messenger RNA (mRNA) expression of PRMT5 was enhanced in the lung tissue specimen of patients with COPD. ${ }^{12}$ However, the role and mechanism of PRMT5 in COPD remains elusive.

In this study, in vitro cell model of COPD was established in human bronchial epithelial cells (16HBE) through incubation with cigarette smoke extract. The effect of PRMT5 on inflammation of cigarette smoke extract-induced $16 \mathrm{HBE}$, as well as the underlying mechanism, was then investigated.

\section{Materials and methods}

\section{Cell culture and treatment and transfection}

Human bronchial epithelial cells (16HBE) were obtained from ATCC (Manassas, VA, USA), and cultured in F12K medium containing $10 \%$ fetal bovine serum (Gibco, Rockville, MD, USA). Cigarette smoke extract was prepared from without filter cigarettes. Briefly, a negative pressure suction device was used to collect cigarette smoke extract from cigarettes without filters. The extract was then poured into a flask for shaking, the bacteria were removed, and the suspensions were collected through a $0.22-\mu \mathrm{m}$ membrane filter. The extract was diluted in $\mathrm{F} 12 \mathrm{~K}$ medium containing $10 \%$ fetal bovine serum to obtain $0.5 \%$, $1 \%$, and $2 \%$ cigarette smoke extracts. The $16 \mathrm{HBE}$ was incubated with $0.5 \%, 1 \%$, and $2 \%$ cigarette smoke extracts for $24 \mathrm{~h}$ before function assays.

\section{Cell viability assay}

The 16HBE post-cigarette smoke extract was plated on a 96-well plate, and transfected with small interfering RNA (siRNA) targeting PRMT5 (si-PRMT5) or the negative control (si-NC) (YouBio, Changsha, China) by Lipofectamine 2000 (Invitrogen, Carlsbad, CA, USA) for 24h. pcDNA-CXCL10 and negative control (pcDNA) were constructed by YouBio, and cotransfected with si-PRMT5 or si-NC into $16 \mathrm{HBE}$ postcigarette smoke extract by Lipofectamine 2000 (Invitrogen) for $24 \mathrm{~h}$. The $16 \mathrm{HBE}$ post-cigarette smoke extract with or without transfection was seeded on plate for another 24, 48, or $72 \mathrm{~h}$. Cells were incubated with MTT solution (Dojindo, Tokyo, Japan) for $4 \mathrm{~h}$, and dissolved with DMSO (dimethyl sulfoxide). Absorbance at $490 \mathrm{~nm}$ was measured by microplate reader (Bio-Rad, Hercules, CA, USA).

\section{Enzyme-linked-immunosorbent serologic assay (ELISA)}

The $16 \mathrm{HBE}$ post-indicated treatment was lysed in radioimmunoprecipitation assay lysis buffer (Beyotime, Shanghai, China) and centrifuged at $12000 \mathrm{~g}$ for $1 \mathrm{~h}$ to collect supernatants. Interlukin (IL)-6, IL-8, tumor necrosis factor- $\alpha$ (TNF- $\alpha$ ), and IL-1 $\beta$ levels were determined by commercial kits (Nanjing Jiancheng Bioengineering Institute, Nanjing, China).

\section{Reverse transcription-quantitative polymerase chain reaction (RT-qPCR)}

The 16HBE was lysed by Trizol (Invitrogen) to collect RNAs. The RNAs were used to generate complementary DNAs (CDNA), and RT-qPCR analysis of PRMT5 was determined by SYBR Premix Ex Taq II (Takara, Tokyo, Japan). Glyceraldehyde 3-phosphate dehydrogenase (GAPDH) was used as an endogenous control, and the following primers were used: PRMT5 (forward primer: 5'-CCATGGAACAAAAAC-3' and reverse primer: 5'-GATCCGCCGCCACCA-3') and GAPDH (forward primer: 5'-CCATCTTCCAGGAGCGAGAT-3' and reverse primer: 5'-TGCTGATGATCTTGAGGCTG-3').

\section{Western blotting assay}

Proteins isolated from $16 \mathrm{HBE}$ were separated by SDSPAGE, and transferred to polyvinylidene difluoride (PVDF) membranes (Millipore, Shanghai, China). The membranes were blocked in phosphate-buffered saline solution with a low-concentration detergent solution (PBST) with 5\% nonfat milk, and incubated with primary and secondary antibodies, including anti-PRMT5 (1:2500; Abcam, Cambridge, UK), anti-CXCL10 (1:3000; Abcam), and anti-GAPDH (1:3500; 
Cell signaling, Boston, MA, USA). ECL reagents (Roche, Shanghai, China) was used to detect protein signals, followed by incubation with horseradish peroxidase (HRP)secondary antibody (1:5000; Abcam).

\section{Statistical analysis}

All experimental data were manifested as mean \pm standard deviation (SD). Differences between groups were analyzed by Student's $t$-test or one-way analysis of variance. $\mathrm{P}<0.05$ was considered as statistically significant.

\section{Results}

\section{PRMT5 was up-regulated in cigarette smoke extract-induced $16 \mathrm{HBE}$}

In order to establish cell model of COPD, 16HBE was incubated with different concentrations of cigarette smoke extract for $24 \mathrm{~h}$. Treatment with cigarette smoke extract reduced cell viability of $16 \mathrm{HBE}$ (Figure $1 \mathrm{~A}$ ). Both mRNA (Figure 1B) and protein (Figure 1C) expressions of PRMT5 were up-regulated in cigarette smoke extractinduced $16 \mathrm{HBE}$, suggesting that PRMT5 might be involved in COPD.

(A)

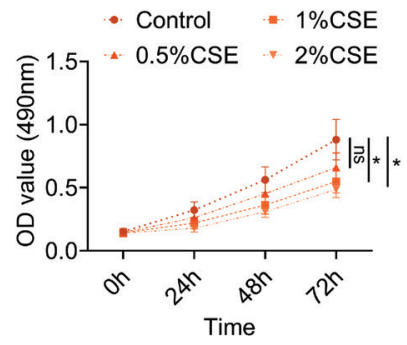

(B)

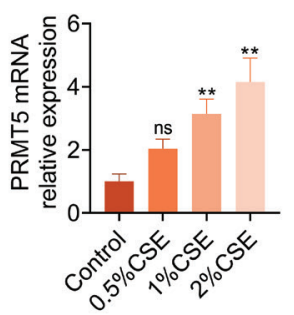

Down-regulation of PRMT5 increased cell viability of cigarette smoke extract-induced $16 \mathrm{HBE}$

Cigarette smoke extract-induced 16HBE was transfected with si-PRMT5 to investigate role of PRMT5 in COPD (Figure 2A). Result established that transfection with si-PRMT5 enhanced cell viability of cigarette smoke extract-induced 16HBE (Figure 2B), demonstrating the proliferative effect of PRMT5 silence on COPD.

\section{Down-regulation of PRMT5 suppressed cigarette smoke extract-induced inflammation in $16 \mathrm{HBE}$}

Levels of IL- 6 , IL- 8 , TNF- $\alpha$, and IL- $1 \beta$ were increased in $16 \mathrm{HBE} 24 \mathrm{~h}$ after treatment with $2 \%$ cigarette smoke extract (Figure 3). However, knockdown of PRMT5 decreased the levels of IL- 6 , IL- 8, TNF- $\alpha$, and IL- $1 \beta$ in cigarette smoke extract-induced 16HBE (Figure 3), indicating the anti-inflammatory effect of PRMT5 silence on COPD.

\section{PRMT5 mediated cell viability and inflammation of cigarette smoke extract-induced $16 \mathrm{HBE}$ through regulation of CXCL10}

The downstream target of PRMT5, CXCL10, was also up-regulated in cigarette smoke extract-induced $16 \mathrm{HBE}$

Figure 1 PRMT5 was up-regulated in cigarette smoke extract-induced 16HBE. (A) Treatment with cigarette smoke extract reduced the cell viability of $16 \mathrm{HBE}$. (B) Treatment with cigarette smoke extract enhanced the mRNA expression of PRMT5 in $16 \mathrm{HBE}$. (C) Treatment with cigarette smoke extract enhanced the protein expression of PRMT5 in $16 \mathrm{HBE}$. *** vs. control, $\mathrm{P}<0.05, \mathrm{P}<0.01$.

(A)

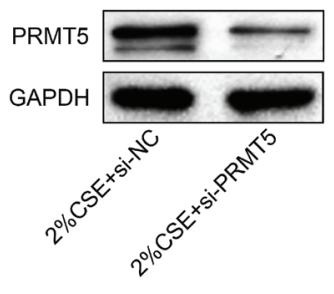

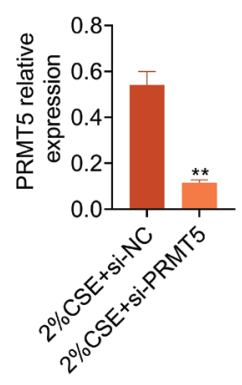

(C)

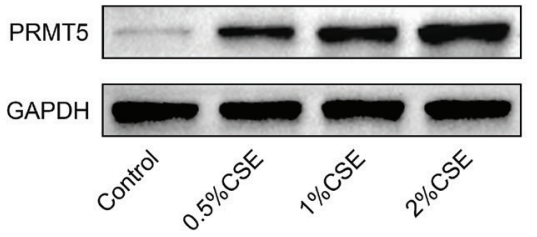

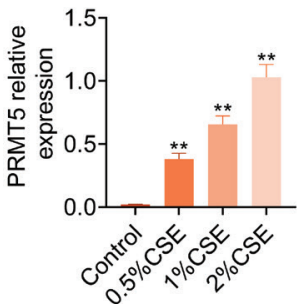

(B)

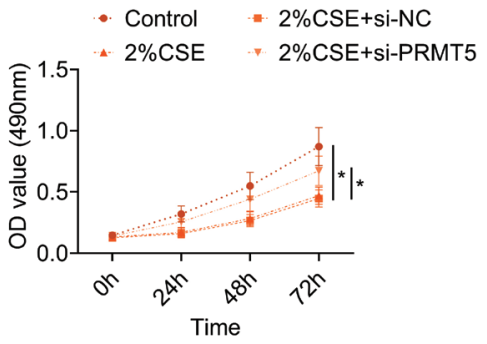

Figure 2 Down-regulation of PRMT5 increased cell viability of cigarette smoke extract-induced 16HBE. (A) Transfection with si-PRMT5 reduced the protein expression of PRMT5 in cigarette smoke extract-induced 16HBE. (B) Transfection with si-PRMT5 promoted cell viability of cigarette smoke extract-induced $16 \mathrm{HBE}$. ${ }^{* * *}$ vs. $2 \% \mathrm{CSE}+$ si-NC, $\mathrm{P}<0.05, \mathrm{P}<0.01$. 

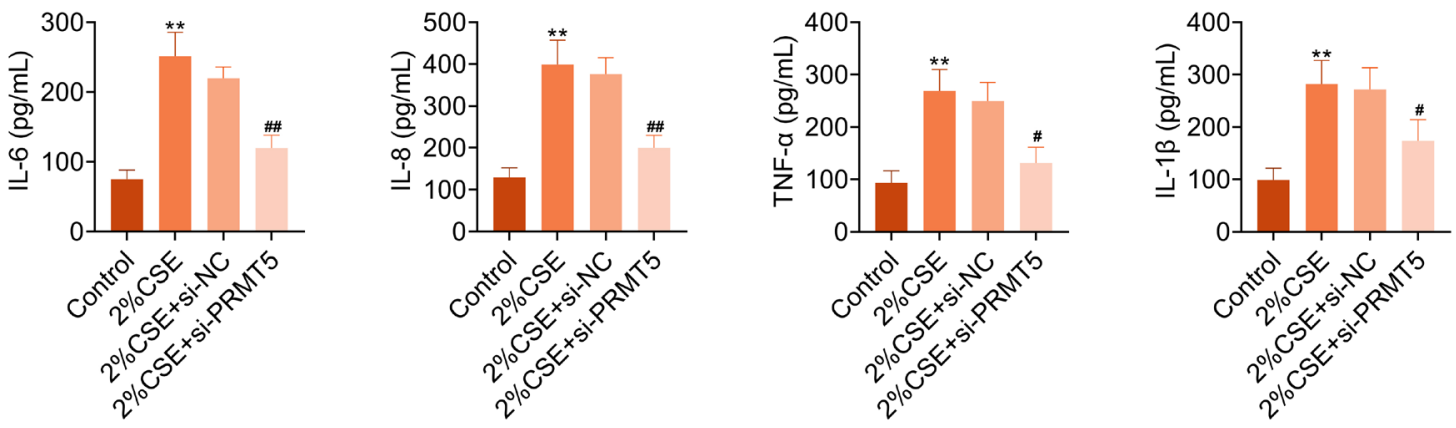

Figure 3 Down-regulation of PRMT5 suppressed cigarette smoke extract-induced inflammation in 16HBE. Knockdown of PRMT5

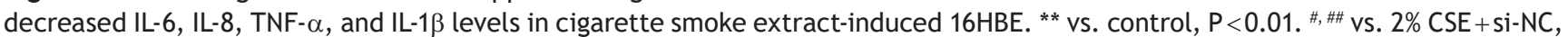
$\mathrm{P}<0.05, \mathrm{P}<0.01$.

(Figure 4A). Knockdown of PRMT5 reduced protein expression of CXCL10 in cigarette smoke extract-induced $16 \mathrm{HBE}$ (Figure 4B). Moreover, over-expression of CXCL10 attenuated PRMT5 silence-induced increase of cell viability in cigarette smoke extract-induced 16HBE (Figure 5A). The reduced IL-6 (Figure 5B), IL-8 (Figure 5C), TNF- $\alpha$ (Figure 5D), and IL-1 $\beta$ (Figure 5E) in cigarette smoke extract-induced $16 \mathrm{HBE}$ transfected with si-PRMT5 were restored by the over-expression of CXCL10, revealing that PRMT5 mediated COPD through regulation of CXCL10.

\section{Discussion}

Metabolism of arginine was altered in patients with COPD, and dysregulated expression or activity of PRMTs was implicated in the pathogenesis of COPD. ${ }^{7}$ For example, cigarette smoke extract induced suppression of PRMT6 to promote
(A)

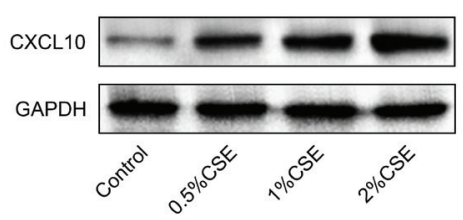

(B)

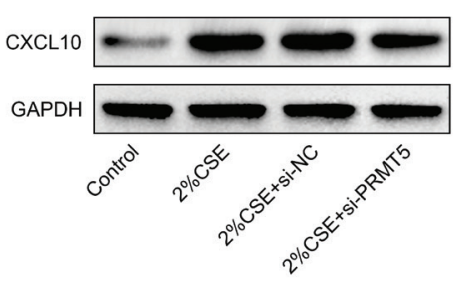

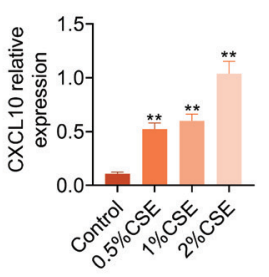

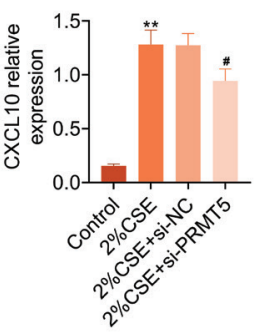

Figure 4 CXCL10 was up-regulated in cigarette smoke extract-induced 16HBE. (A) Treatment with cigarette smoke extract enhanced protein expression of CXCL10 in 16HBE. (B) Knockdown of PRMT5 decreased CXCL10 expression in cigarette smoke extract-induced $16 \mathrm{HBE}$. ${ }^{* *}$ vs. control, $\mathrm{P}<0.01$. \# vs. $2 \%$ CSE + si-NC, $\mathrm{P}<0.05$. airway epithelial cell death. ${ }^{13}$ Degradation of PRMT6 aggravated cigarette smoke extract-induced lung epithelial cell apoptosis and inflammation. ${ }^{14}$ Considering that PRMT5 was associated with lung branching morphogenesis ${ }^{15}$ and enhanced in the lung tissue specimen of patients with COPD,${ }^{12}$ the regulatory role of PRMT5 on COPD was investigated in this study.

Cigarette smoke extract has been shown to induce apoptosis and inflammation of bronchial epithelial cells. ${ }^{16}$ Therefore, cigarette smoke extract-induced 16HBE was widely used as cell model of COPD. ${ }^{16}$ In this study, cigarette smoke extract induced decrease in the cell viability of $16 \mathrm{HBE}$. In line with the previous study that PRMT5 was up-regulated in the lung tissue specimen of patients with COPD, ${ }^{12}$ PRMT5 was also enhanced in 16HBE after treatment with cigarette smoke extract. Moreover, knockdown of PRMT5 increased cell viability of cigarette smoke extract-induced $16 \mathrm{HBE}$, suggesting the proliferative effect of PRMT5 silence on COPD. However, the role of PRMT5 on cigarette smoke extract-induced apoptosis of $16 \mathrm{HBE}$ should be investigated in the future research.

Cigarette smoke promotes activation of peripheral blood leukocytes and release of proinflammatory mediators resulting in tissue damage in pulmonary tissues during COPD progression. ${ }^{17}$ Suppression of inflammatory responses was considered to be a potential therapeutic strategy for the prevention of COPD.(17) Proinflammatory mediators (IL$1 \beta$, IL-6, and TNF- $\alpha$ ) were up-regulated in cigarette smoke extract-induced $16 \mathrm{HBE} .{ }^{16} \mathrm{IL}-6, \mathrm{IL}-8, \mathrm{TNF}-\alpha$, and IL-1 $\beta$ levels in this study were found to be up-regulated in cigarette smoke extract-induced 16HBE. Levels of IL-1 $\beta$ and TNF- $\alpha$ in fibroblast-like synoviocytes were reduced by inhibition of PRMT5 to attenuate rheumatoid arthritis. ${ }^{11}$ This study also confirmed the anti-inflammatory effect of PRMT5 silence on COPD, as evidenced that knockdown of PRMT5 decreased the levels of IL- 6 , IL- 8 , TNF- $\alpha$, and IL- $1 \beta$ in cigarette smoke extract-induced $16 \mathrm{HBE}$. Moreover, cigarette smoke has also been shown to promote secretion of reactive oxygen species and deplete antioxidants, shift the balance to oxidant burden, and aggravate tissue damage. ${ }^{17}$ The increased alveolar macrophages and inflammatory leukocytes promote the pro-oxidant environment of the pulmonary tissues in COPD patients. ${ }^{17}$ Since inhibition of PRMT5 suppressed oxidative stress in a renal ischemia-reperfusion injury model, ${ }^{18}$ 
(A)

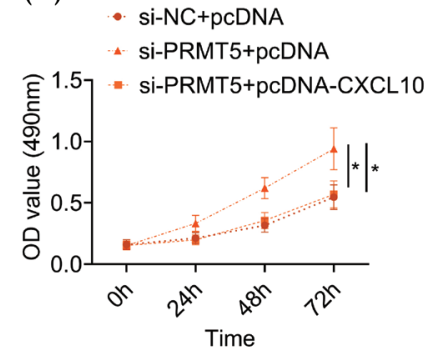

(B)

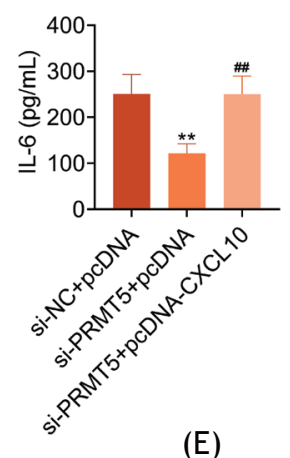

(C)

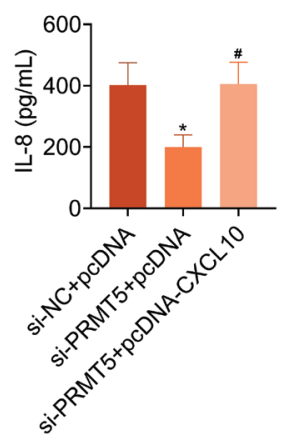

(D)

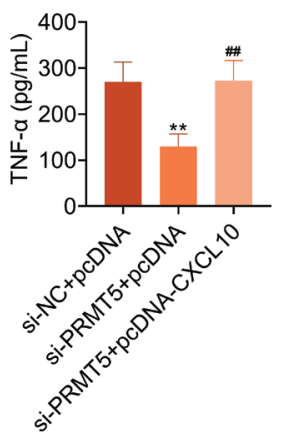

(E)

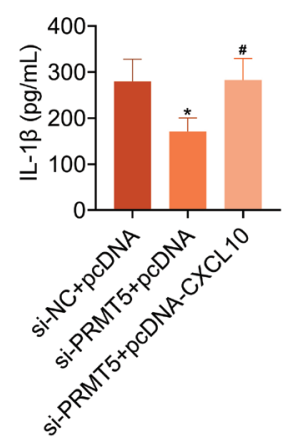

Figure 5 PRMT5-mediated cell viability and inflammation of cigarette smoke extract-induced 16HBE through regulation of CXCL10. (A) Over-expression of CXCL10 attenuated PRMT5 silence-induced increase of cell viability in cigarette smoke extract-induced 16HBE. (B) Over-expression of CXCL10 attenuated PRMT5 silence-induced decrease of IL-6 in cigarette smoke extract-induced 16HBE. (C) Over-expression of CXCL10 attenuated PRMT5 silence-induced decrease of IL- 8 in cigarette smoke extract-induced 16HBE. (D) Over-expression of CXCL10 attenuated PRMT5 silence-induced decrease of TNF- $\alpha$ in cigarette smoke extract-induced 16HBE. (E) Over-expression of CXCL10 attenuated PRMT5 silence-induced decrease of IL-1 $\beta$ in cigarette smoke extract-induced 16HBE. *** vs. control, $\mathrm{P}<0.05, \mathrm{P}<0.01 . \#, \#$ vs. $2 \% \mathrm{CSE}+$ si-NC, $\mathrm{P}<0.05, \mathrm{P}<0.01$.

the anti-oxidant effects of PRMT5 silence on COPD must be investigated in the future research.

Chemokines mediate recruitment of immune cells into inflammatory lesions, and are related to the development of COPD. ${ }^{19} \mathrm{~A}$ previous study has demonstrated that CXCL10 could recruit CXCR3+ type-1 T-lymphocytes to the peripheral airways of smokers with COPD, and type-1 T-lymphocytes express interferon gamma to promote activation of macrophages, thus releasing inflammatory cytokines, including IL-6, IL-8, TNF- $\alpha$, and IL-1 $\beta$.(20) Therefore, increased serum CXCL10 was regarded as a biomarker of COPD. ${ }^{21}$ Suppression of CXCL10 attenuated inflammatory response in cigarette smoke-induced COPD. ${ }^{22,23} \mathrm{~A}$ past study has established that PRMT5 mediated methylation of NF- $\kappa$ B p65 and regulated expression of CXCL10 in endothelial cells. ${ }^{24}$ Repression of PRMT5 reduced CXCL10 expression to suppress osteoclast differentiation and attenuated ovariectomy-induced bone loss. ${ }^{25}$ CXCL10 was found to be up-regulated in cigarette smoke extract-induced $16 \mathrm{HBE}$, and knockdown of PRMT5 decreased the protein expression of CXCL10. Moreover, over-expression of CXCL10 attenuated PRMT5 silence-induced increase of cell viability and decrease of IL- 6, IL- 8 , TNF- $\alpha$, and IL- $1 \beta$ in cigarette smoke extract-induced $16 \mathrm{HBE}$, suggesting that PRMT5CXCL10 axis was implicated in the pathogenesis of COPD.

\section{Conclusion}

In summary, PRMT5 and the downstream target were differentially expressed in cigarette smoke extract-induced $16 \mathrm{HBE}$, and knockdown of PRMT5 suppressed inflammatory response in cigarette smoke extract-induced $16 \mathrm{HBE}$ through down-regulation of CXCL10. This study provided a novel target for clinical treatment of COPD. However, the protective effect of PRMT5-CXCL10 against COPD must be confirmed in murine emphysema model induced by cigarette smoke extract.

\section{Competing interests}

No conflict of interest to disclose by authors.

\section{Contribution of authors}

Junfang Ju and Yinghe Chun designed the study, supervised data collection, analyzed and interpreted the data, and prepared and reviewed the final draft of the manuscript for publication. Both authors read and approved the final manuscript. 


\section{References}

1. MacNee W. Pathogenesis of chronic obstructive pulmonary disease. Proc Am Thorac Soc. 2005;2(4):258-91. https://doi. org/10.1513/pats.200504-045SR

2. López-Campos JL, Tan W, Soriano JB. Global burden of COPD. Respirology. 2016;21(1):14-23. https://doi.org/10.1111/ resp. 12660

3. Jindal SK. Chronic obstructive pulmonary disease in non-smokers-Is it a different phenotype? Indian J Med Res. 2018;147(4):337-9. https://doi.org/10.4103/ijmr.IJMR_10_18

4. Barnes PJ. Inflammatory mechanisms in patients with chronic obstructive pulmonary disease. J Allergy Clin Immunol. 2016;138(1):16-27. https://doi.org/10.1016/j.jaci.2016.05.011; https://doi.org/10.1016/j.jaci.2009.11.008

5. Roda MA, Xu X, Abdalla TH, Sadik M, Szul T, Bratcher PE, et al. Proline-glycine-proline peptides are critical in the development of smoke-induced emphysema. Am J Respir Cell Mol Biol. 2019;61(5):560-6. https://doi.org/10.1165/rcmb.2018-02160C

6. Wu Q, Schapira M, Arrowsmith CH, Barsyte-Lovejoy D. Protein arginine methylation: From enigmatic functions to therapeutic targeting. Nature Rev Drug Discov. 2021;20(7):509-30. https://doi.org/10.1038/s41573-021-00159-8

7. Zakrzewicz D, Zakrzewicz A, Preissner KT, Markart $P$, Wygrecka M. Protein arginine methyltransferases (PRMTs): Promising targets for the treatment of pulmonary disorders. Int J Mol Sci. 2012;13(10):12383-400. https://doi.org/10.3390/ ijms131012383

8. Li M, An W, Xu L, Lin Y, Su L, Liu X. The arginine methyltransferase PRMT5 and PRMT1 distinctly regulate the degradation of anti-apoptotic protein CFLAR(L) in human lung cancer cells. J Exp Clin Cancer Res. 2019;38(1):Art. No. 64. https://doi. org/10.1186/s13046-019-1064-8

9. Jing P, Zhao N, Ye M, Zhang Y, Zhang Z, Sun J, et al. Protein arginine methyltransferase 5 promotes lung cancer metastasis via the epigenetic regulation of miR-99 family/FGFR3 signaling. Cancer Lett. 2018;427:38-48. https://doi.org/10.1016/j. canlet.2018.04.019

10. Kim JH, Yoo BC, Yang WS, Kim E, Hong S, Cho JY. The role of protein arginine methyltransferases in inflammatory responses. Mediators Inflamm. 2016;2016:4028353. https:// doi.org/10.1155/2016/4028353

11. Chen $\mathrm{D}$, Zeng $\mathrm{S}$, Huang $\mathrm{M}$, $\mathrm{Xu} \mathrm{H}$, Liang $\mathrm{L}$, Yang $\mathrm{X}$. Role of protein arginine methyltransferase 5 in inflammation and migration of fibroblast-like synoviocytes in rheumatoid arthritis. J Cell Mol Med. 2017;21(4):781-90. https://doi.org/10.1111/jcmm.13020

12. Yildirim A, Königshoff $M$, Wang $Q$, Eickelberg $O$, editors. Expression profiling of protein arginine methyltransferase (Prmt) isoforms in chronic obstructive pulmonary disease (COPD). Am J Resp Crit Care Med. 2010;181:A4954. https:// doi.org/10.1164/ajrccm-conference.2010.181.1

13. Li T, Fanning KV, Nyunoya T, Chen Y, Zou C. Cigarette smoke extract induces airway epithelial cell death via repressing PRMT6/AKT signaling. Aging (Albany NY). 2020;12(23):2430117. https://doi.org/10.18632/aging.202210

14. Li T, He X, Luo L, Zeng H, Ren S, Chen Y. F-Box protein FBXW17mediated proteasomal degradation of protein methyltransferase
PRMT6 exaggerates CSE-induced lung epithelial inflammation and apoptosis. Front Cell Dev Biol. 2021;9:599020. https://doi. org/10.3389/fcell.2021.599020

15. Li Q, Jiao J, Li H, Wan H, Zheng C, Cai J, et al. Histone arginine methylation by Prmt5 is required for lung branching morphogenesis through repression of BMP signaling. J Cell Sci. 2018 July;131(14):jcs217406. https://doi.org/10.1242/ jcs. 217406

16. Shen $Q$, Zheng J, Wang $X$, Hu W, Jiang $Y$, Jiang $Y$. LncRNA SNHG5 regulates cell apoptosis and inflammation by miR-132/ PTEN axis in COPD. Biomed Pharmacother. 2020;126:110016. https://doi.org/10.1016/j.biopha.2020.110016

17. Garcia-Rio F, Miravitlles M, Soriano JB, Muñoz L, DuranTauleria E, Sánchez G, et al. Systemic inflammation in chronic obstructive pulmonary disease: A population-based study. Resp Res. 2010;11(1):63. https://doi.org/10.1186/1465-9921-11-63

18. Diao C, Chen Z, Qiu T, Liu H, Yang Y, Liu X, et al. Inhibition of PRMT5 attenuates oxidative stress-induced pyroptosis via activation of the Nrf2/HO-1 signal pathway in a mouse model of renal ischemia-reperfusion injury. Oxid Med Cell Longev. 2019;2019:2345658. https://doi.org/10.1155/2019/2345658

19. Hao W, Li M, Pang Y, Du W, Huang X. Increased chemokines levels in patients with chronic obstructive pulmonary disease: Correlation with quantitative computed tomography metrics. Br J Radiol. 2020;94(1118):20201030. https://doi.org/10.1259/ bjr.20201030

20. Saetta M, Mariani M, Panina-Bordignon P, Turato G, Buonsanti $C$, Baraldo $S$, et al. Increased expression of the chemokine receptor CXCR3 and its ligand CXCL10 in peripheral airways of smokers with chronic obstructive pulmonary disease. Am J Resp Crit Care Med. 2002;165(10):1404-9. https:// doi.org/10.1164/rccm.2107139

21. Maurer JR. Acute exacerbations of chronic obstructive pulmonary disease: Identification of biologic clusters and their biomarkers. In: 2012 Yearbook of pulmonary disease. Philedelphia, PA: Elsevier; 2012;, Chap 2, pp. 42-3. https:// doi.org/10.1016/j.ypdi.2012.01.014

22. Jing $\mathrm{H}$, Liu L, Zhou J, Yao H. Inhibition of C-X-C motif chemokine 10 (CXCL10) protects mice from cigarette smoke-induced chronic obstructive pulmonary disease. Med Sci Monit. 2018;24:5748-53. https://doi.org/10.12659/MSM.909864

23. Hardaker EL, Bacon AM, Carlson K, Roshak AK, Foley JJ, Schmidt DB, et al. Regulation of TNF- $\alpha$ and IFN- $\gamma$ induced CXCL10 expression: Participation of the airway smooth muscle in the pulmonary inflammatory response in chronic obstructive pulmonary disease. FASEB J. 2004;18(1):191-3. https:// doi.org/10.1096/fj.03-0170fje

24. Harris DP, Bandyopadhyay S, Maxwell TJ, Willard B, DiCorleto PE. Tumor necrosis factor (TNF)- $\alpha$ induction of CXCL10 in endothelial cells requires protein arginine methyltransferase 5 (PRMT5)-mediated nuclear factor (NF)- $\mathrm{kB}$ p65 methylation. J Biol Chem. 2014;289(22):15328-39. https://doi. org/10.1074/jbc.M114.547349

25. Dong $\mathrm{Y}$, Song C, Wang $\mathrm{Y}$, Lei Z, Xu F, Guan H, et al. Inhibition of PRMT5 suppresses osteoclast differentiation and partially protects against ovariectomy-induced bone loss through downregulation of CXCL10 and RSAD2. Cell Signal. 2017;34:55-65. https://doi.org/10.1016/j.cellsig. 2017.03.004 